\title{
ALS patients' regulatory T lymphocytes are dysfunctional, and correlate with disease progression rate and severity
}

\author{
David R. Beers, ${ }^{1}$ Weihua Zhao, ${ }^{1}$ Jinghong Wang, ${ }^{1}$ Xiujun Zhang, ${ }^{1}$ Shixiang Wen, ${ }^{1}$ Dan Neal, ${ }^{2}$ \\ Jason R. Thonhoff, ${ }^{1}$ Abdullah S. Alsuliman, ${ }^{3}$ Elizabeth J. Shpall, ${ }^{3}$ Katy Rezvani, ${ }^{3}$ \\ and Stanley H. Appel ${ }^{1}$ \\ 1Peggy and Gary Edwards ALS Laboratory, Department of Neurology, Houston Methodist Neurological Institute, Houston \\ Methodist Research Institute, Houston Methodist Hospital, Houston, Texas, USA. ${ }^{2}$ Department of Surgery, University \\ of Florida, Gainesville, Florida, USA. ${ }^{3}$ Department of Stem Cell Transplant and Cellular Therapy, University of Texas MD \\ Anderson Cancer Center, Houston, Texas, USA.
}

\begin{abstract}
Neuroinflammation is a pathological hallmark of ALS in both transgenic rodent models and patients, and is characterized by proinflammatory $T$ lymphocytes and activated macrophages/ microglia. In ALS mouse models, decreased regulatory T lymphocytes (Tregs) exacerbate the neuroinflammatory process, leading to accelerated motoneuron death and shortened survival; passive transfer of Tregs suppresses the neuroinflammation and prolongs survival. Treg numbers and FOXP3 expression are also decreased in rapidly progressing ALS patients. A key question is whether the marked neuroinflammation in ALS can be attributed to the impaired suppressive function of ALS Tregs in addition to their decreased numbers. To address this question, $T$ lymphocyte proliferation assays were performed. Compared with control Tregs, ALS Tregs were less effective in suppressing responder T lymphocyte proliferation. Although both slowly and rapidly progressing ALS patients had dysfunctional Tregs, the greater the clinically assessed disease burden or the more rapidly progressing the patient, the greater the Treg dysfunction. Epigenetically, the percentage methylation of the Treg-specific demethylated region was greater in ALS Tregs. After in vitro expansion, ALS Tregs regained suppressive abilities to the levels of control Tregs, suggesting that autologous passive transfer of expanded Tregs might offer a novel cellular therapy to slow disease progression.
\end{abstract}

Conflict of interest: The authors have declared that no conflict of interest exists.

Submitted: July 12, 2016 Accepted: January 19, 2017 Published: March 9, 2017

Reference information: JCI Insight. 2017;2(5):e89530. https:// doi.org/10.1172/jici.insight.89530.

\section{Introduction}

Neuroinflammation is a hallmark of many neurodegenerative diseases and is a significant component of the pathology of ALS, a disorder that selectively destroys upper and lower motoneurons, resulting in death. ALS is a heterogeneous disorder with differing rates of progression and lengths of disease, and although neuroinflammation is a pathological feature, its role in the pathogenic process is still unclear $(1,2)$. Multiple studies have addressed T lymphocyte infiltration in the CNS of patients with ALS, possibly related to the increased chemokine ( $\mathrm{C}-\mathrm{C}$ motif) ligand 2 (CCL2) levels, and the morphological activation of microglia (3-5); lymphocytes were found in the majority of patient spinal cords and along the vessels in the precentral gyrus extending into the areas of neuronal injury (6-10). Our investigations found perivascular and intraparenchymal $\mathrm{CD} 4^{+} \mathrm{T}$ lymphocytes in the proximity of degenerating corticospinal tracts and ventral horns in two-thirds of ALS patients (11). In the blood of patients with ALS, alterations in T lymphocyte populations have also been described as compared with controls (12-16); however, these studies do not address whether $\mathrm{T}$ lymphocytes directly or indirectly influence disease progression.

The innate and adaptive immune systems play pivotal and interdependent roles in regulating the rate of disease progression $(2,5,17-22)$. In the ALS model of mice overexpressing mutant superoxide dismutase 1 (mSOD1), $\mathrm{CD}^{+} \mathrm{T}$ lymphocytes slowed disease progression, increased disease duration by $50 \%$, modified the microglial phenotypes, and extended survival $(19,20,22)$. One population of $\mathrm{T}$ lymphocytes, the $\mathrm{CD}^{+}{ }^{+} \mathrm{CD} 25^{\text {hiFoxP }} 3^{+}$regulatory $\mathrm{T}$ lymphocytes (Tregs), were found to be increased at early, slowly progressing stages, and decreased during the rapidly progressing phase, possibly through the loss of FoxP3 expression 
in Tregs; the suppressive effects of Tregs on the adaptive and innate immune systems have been documented $(19,23-26)$. Passive transfer of endogenous Tregs from ALS mice in the early disease stage into recipient ALS mice, without ex vivo activation, were also shown to sustain IL-4 levels and M2 microglia, lengthen disease duration, and prolong survival $(19,20)$.

Tregs suppress both innate and adaptive immune reactions detrimental to the host, downregulate proinflammatory cytokine production, and can suppress the activation/expansion of $\mathrm{CD} 4^{+} \mathrm{CD} 25^{-}$effector $\mathrm{T}$ lymphocytes (Teffs). The passive transfer of ex vivo-activated Tregs was shown to suppress toxic microglial responses, upregulate glial cell-derived neurotrophic factor (CDNF) and TGF- $\beta$, and protected neurons in a model of Parkinson's disease $(27,28)$. Recently, Tregs have been shown to directly steer the differentiation of macrophages and microglia toward an alternative M2 activation state (29-33), and in turn, M2 cells induce Tregs which suppress Teffs $(31,34-36)$. Treg-mediated suppression involves multicellular clusters consisting of responder T lymphocytes (Tresps), antigen-presenting cells, and membrane-bound and/or soluble inhibitory molecules. Tregs suppress $\mathrm{T}$ lymphocyte proliferation, downregulate proinflammatory cytokine production (IFN- $\gamma$ and TNF- $\alpha$ ), and directly inhibit IL-2 mRNA transcription. Therefore, Tregs are critically involved in suppressing inflammation induced by neurotoxic $\mathrm{T}$ lymphocytes and microglia/ macrophages, and since they play a prominent role in slowing the rate of progression in ALS mice, they are directly involved in modifying disease progression $(17,19,20,22)$.

In blood leukocytes isolated from patients with rapidly progressing ALS, both numbers of Tregs and their FOXP3 protein expression were reduced, and these levels inversely correlated with rates of disease progression (37). The mRNA levels of FOXP3, TGF- $\beta$, IL-4, and GATA-3, a Th2 transcription factor, were reduced in rapidly progressing patients and inversely correlated with progression rates; both FOXP3 and GATA3 were accurate indicators of progression rates. No differences in IL-10, TBX21 (a Th1 transcription factor), or IFN- $\gamma$ expression were found between slow and rapidly progressing patients. Furthermore, receiver operating characteristic (ROC) analyses demonstrated that FOXP3 expression accurately reflected the progression rates of ALS patients from all stages of disease at the time of leukocyte collection. Thus, decreased FOXP3 levels in leukocytes during early phases of ALS may be a measure of disease progression rates and of predicted reduced survival.

In summary, data collected from ALS patients and mSOD1 mice, a model of human ALS, suggest that reduced Treg numbers and reduced FOXP3 mRNA levels are correlated with disease progression rates and decreased survival. However, it was still unknown whether the suppressive functions of Tregs from patients with ALS were compromised. Do ex vivo Tregs from patients with ALS lack suppressive capabilities compared with Tregs from healthy control volunteers, and if so, does the lack of suppression correlate with disease burden and rates of progression in these patients with ALS?

\section{Results}

Contamination with non-Tregs in the isolated Treg population. The purity of the Tregs was analyzed by flow cytometry. The percentage of $\mathrm{CD} 4{ }^{+} \mathrm{CD} 25^{\mathrm{hi}}$ Tregs was equivalent between ALS patients and healthy volunteer controls (82\%), and expressed variable levels of FOXP3. Additional analyses of the positively selected Tregs obtained from the patients with ALS and healthy controls that were subsequently used in these studies revealed that the number of $\mathrm{CD}^{+} \mathrm{CD} 4{ }^{+} \mathrm{CD} 25^{\mathrm{hi}} \mathrm{CD} 127^{+}$cells represented $3 \%$ (ALS, $2.8 \% \pm 0.34[n=3]$; healthy controls, $3.3 \% \pm 0.30[n=3] ; P=0.33$ ) of the total $\mathrm{CD} 3^{+} \mathrm{CD} 4^{+} \mathrm{CD} 25^{\text {hi }} \mathrm{CD} 127^{\text {lo }}$ (Tregs) cells. Furthermore, this low number of $\mathrm{CD}^{+} \mathrm{CD}^{+} \mathrm{CD} 25^{\text {hi }} \mathrm{CD} 127^{+}$cells was similar between slowly and rapidly progressing ALS patients (slowly progressing, 2.6\% $\pm 0.20[n=3]$; rapidly progressing, $2.8 \% \pm 0.30[n=3] ; P=$ 0.73 ). These results are consistent with a previous study that found the frequencies of $C D 4^{+} C D 25^{\text {hi }} C D 127^{\text {lo }}$ naturally occurring Tregs in patients with ALS and healthy controls were also not different (38).

Treg suppression of Tresp proliferation. The Tregs from ALS patients were next evaluated for their ability to suppress the proliferation of their corresponding CD4 ${ }^{+} \mathrm{CD} 25^{-}$Tresps and compared with control Tresp/Treg cocultures. Patients with ALS and the healthy volunteer controls were age and gender matched (Supplemental Table 1). In addition, none of the patients with ALS had a family history of ALS, nor did they have evidence of genetic mutations, even in the absence of a family history. However, $10 \%$ of patients with sporadic ALS have C9orf72 hexanucleotide repeats, but there was no evidence of any mutant genes in the sporadic patients studied. Furthermore, all patients were diagnosed with definite or probable sporadic ALS according to revised El Escorial criteria of the World Federation of Neurology (39). These studies were performed with IRB approval, and all patients with ALS and 

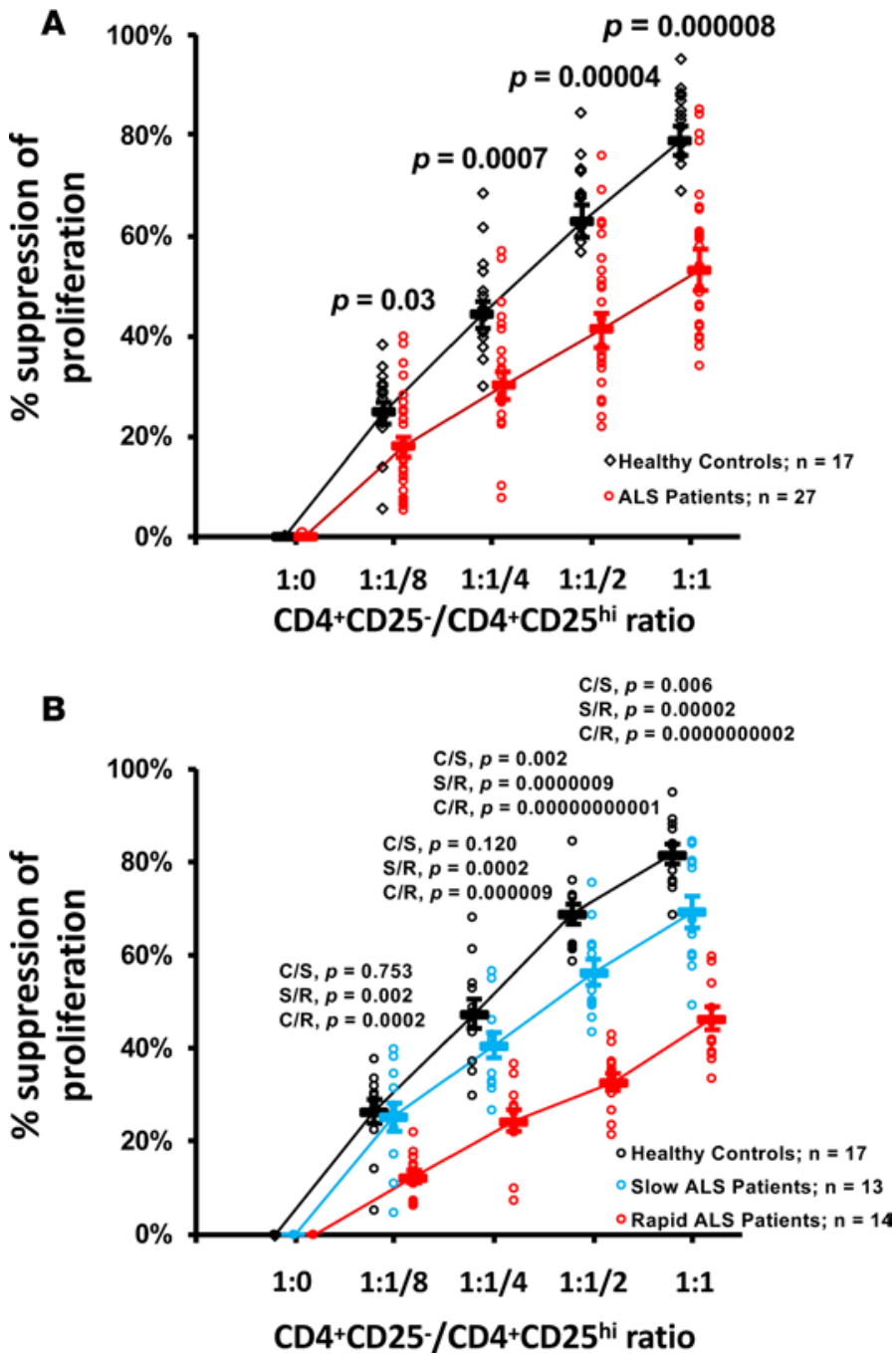

Figure 1. Tregs from ALS patients are dysfunctional. (A) Scatter plot of the Treg suppression assay results using Tregs from individual controls and ALS patients. Tregs from patients with ALS are not as functionally suppressive as Tregs from control individuals. Significance determined by Student's $t$ test. (B) Tregs from patients with slowly progressing ALS and the control individuals are more suppressive than patients with rapidly progressing ALS across all responder T lymphocyte/Treg suppression assay ratios (CD4 ${ }^{+} C D 25^{-} / C D 4^{+} C D 25^{\text {hi }}$ ). C, control; S, slowly progressing ALS patients; R, rapidly progressing ALS patients.

healthy volunteer controls signed a written informed consent, and in a non-blinded manner.

In these studies, we varied the number of Tregs and kept the number of Tresps constant. Although variable, the Tresps from each ALS patient, and those of the healthy controls, expanded to a similar extent (data not shown). We found that Tregs from patients with ALS exhibited reduced suppressive capacity on their corresponding Tresps across the entire range of Tresp/Treg ratios (Figure 1A). Plots of the individual patients from both groups showed a tight linear relationship between percentage of suppression and increasing ratio. Percentage of suppression also increased more in the control group compared with the ALS group. Using a mixed-effects linear model to evaluate the effects of group and ratio on percentage of suppression, both ratio and group were associated with percentage of suppression $(P=0.0001$ for the interaction term). For the ALS group, percentage of suppression increased 11.7 points with each level increase in ratio (95\% CI $=[8.17,15.26], P<0.0001)$. For the control group, the percentage of suppression increased 18.2 points with each level increase in ratio $(95 \% \mathrm{CI}=[15.08,21.26], P<0.0001)$. Across all measurements, the control group had values that were on average 6.9 points higher than the ALS group $(95 \% \mathrm{CI}=[0.476,13.37]$, $P=0.036$ ), and across both groups, percentage of suppression increased 14.2 points with each level increase in ratio $(95 \% \mathrm{CI}=$ $[12.43,15.99], P<0.0001)$. Thus, the percentage of suppression increased with ratio for both groups $(P<$ $0.0001)$, but increased more for the control group $(P=0.0001)$, and on average, the control group had a higher percentage of suppression across all ratios compared with the ALS group $(P=0.036)$.

Slowly progressing patients' Tregs are more suppressive. When the ALS patients were subdivided into slow and rapid progression rates, as previously established (slow progression, < 1.5 Appel ALS [AALS] points/month; rapid progression, $\geq 1.5$ AALS points/month) (37), the slowly progressing ALS patients' and control group's Tregs were found to be more suppressive than the rapidly progressing ALS patients' Tregs across all Tresp/ Treg ratios (Figure 1B). Again, using a mixed-effects linear model to evaluate the effects of group and ratio on percentage of suppression, both ratio and group were associated with percentage of suppression $(P=0.0001$ for the interaction term). Across all measured points, the rapidly progressing ALS patients had values that were on average 26.7 points lower than the control group (95\% $\mathrm{CI}=[-32.4,-21.0], P<0.0001)$, and 18.7 points below slowly progressing ALS patients $(95 \% \mathrm{CI}=[-24.3,-13.0], P<0.0001)$. Also, across all measured points, the slowly progressing ALS patients had values that were on average 8.1 points lower than the control group (95\% CI $=[-13.8,-2.27], P=0.008)$, and that percentage of suppression increased 14.7 points with each level increase in ratio $(95 \% \mathrm{CI}=[13.2,16.3], P<0.0001)$. In addition, rapidly progressing ALS patients' Tregs were different than Tregs from controls across all Tresp/Treg ratios $(P<0.001$ at all ratios). Interestingly, the means plot showed a relatively small difference between the Tregs from slowly progressing ALS patients and the control group, but both groups exhibited a greater percentage of suppression than the Tregs from rapidly progressing ALS patients. These data suggested that ex vivo ALS patient Tregs were dysfunctional compared with Tregs obtained from the control group. While both slowly and rapidly progressing ALS patients had dysfunctional Tregs, the suppressive dysfunction was most marked in Tregs from rapidly progressing ALS patients. 


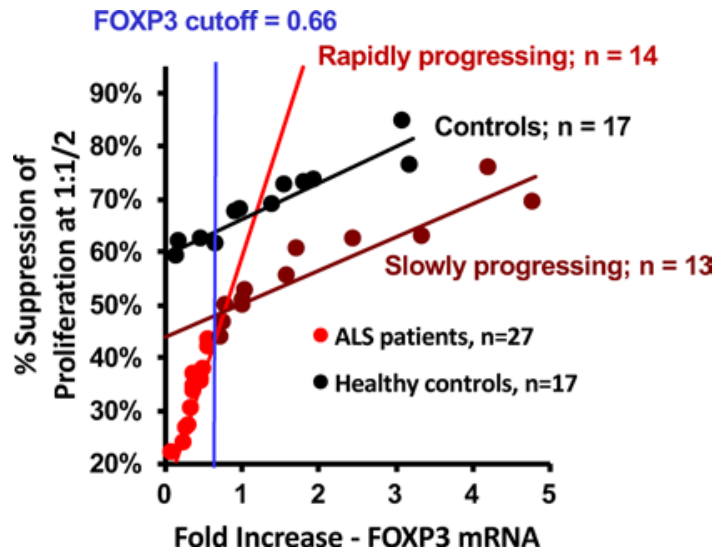

Figure 2. The correlation of Treg suppression versus FOXP3 mRNA expression levels. The percentage Treg suppression of responder T lymphocyte (Tresp) proliferation at the 1:1/2 Tresp/Treg ratio was very strongly correlated with FOXP3 mRNA expression.

Treg suppression correlates with FOXP3 expression. Since we determined that low FOXP3 levels in leukocytes from ALS patients obtained early in the disease accurately predicted future rapid progression rates and more importantly, low FOXP3 levels predicted reduced survival, the FOXP3 expression in leukocytes was correlated with the same patient's Tregs' suppressive function (37). At the 1:1/2 Tresp/Treg ratio, the percentage of suppression was very strongly correlated with FOXP3 expression for control volunteers $(\mathrm{R}=0.945$, $P<0.001$; Figure 2), slowly progressing ALS patients $(\mathrm{R}=0.935, P<0.001)$, and rapidly progressing ALS patients $(\mathrm{R}=0.941, P<0.001)$. Thus, confirming our previously published ROC analysis cutoff for FOXP3 mRNA expression relative to the control group for predictability ( 0.66 , see Figure $1 C)$, the rapidly progressing ALS patients fell below this cutoff (37). Although most of the slowly progressing ALS patients were above this cutoff, their Tregs lacked suppressive abilities. Additionally, for any given FOXP3 expression level, the suppressive function of ALS Tregs was decreased compared with the control group Tregs.

Treg suppressive deficiency correlates with disease burden and rate of disease progression. We also determined that ALS patients with low FOXP3 expression levels had increased impairment (disease burden) compared with patients with high FOXP3 levels. Slowly progressing ALS patients had an AALS score of $57.54 \pm$ 3.22 points $(n=13)$, whereas the rapidly progressing patients had a score of $90.91 \pm 7.83$ points $(n=14)$ : $P=0.001$. We chose these patients based on our previously published human and mouse data $(3,4,7)$. In the human study, we showed that both numbers of Tregs and their FOXP3 protein expression levels were reduced in rapidly progressing ALS patients and inversely correlated with progression rates. Low FOXP3 was also correlated with reduced survival. In our animal studies using Tregs obtained from G93A SOD1 ALS (mSOD1) mice, a model of human ALS, we found that the passive transfers of Tregs obtained from mSOD1 mice in the late stage of disease had no effect on slowing the rate of disease progression in earlystage recipient mSOD1 mice. Furthermore, Tregs obtained from mSOD1 mice had no effect on slowing the rate of disease progression when the ALS mice were in the late phase of their disease. When the burden of disease (AALS points) at blood draw was compared with the 1:1/2 Tresp/Treg ratio for the percentage of suppression of Tresp proliferation, there was a correlation $(\mathrm{R}=0.529, P=0.008$; Figure $3 \mathrm{~A})$. Therefore, although the disease burden of the patients fell into 2 populations, the Treg dysfunction correlated inversely over the entire range of disease burdens. In addition, when the rate of disease progression (AALS points/month) at blood draw was compared with the 1:1/2 Tresp/ Treg ratio for the percentage of suppression of proliferation, there again was a correlation $(\mathrm{R}=0.534$, $P=0.006$; Figure 3B). Thus, the Treg dysfunction negatively correlated with both the burden of disease and the rate of disease progression; the more

Figure 3. Treg suppressive deficiency correlates with disease burden and rate of disease progression. (A) There is a correlation between burden of disease (Appel ALS [AALS] points) at blood draw and the level of Treg suppression of responder T lymphocyte (Tresp) proliferation at the 1:1/2 Tresp/Treg ratio. (B) There is a correlation between the rates of disease progression (AALS points/month) at blood draw and Treg suppression of Tresp proliferation. $P$ values obtained by Pearson correlation.
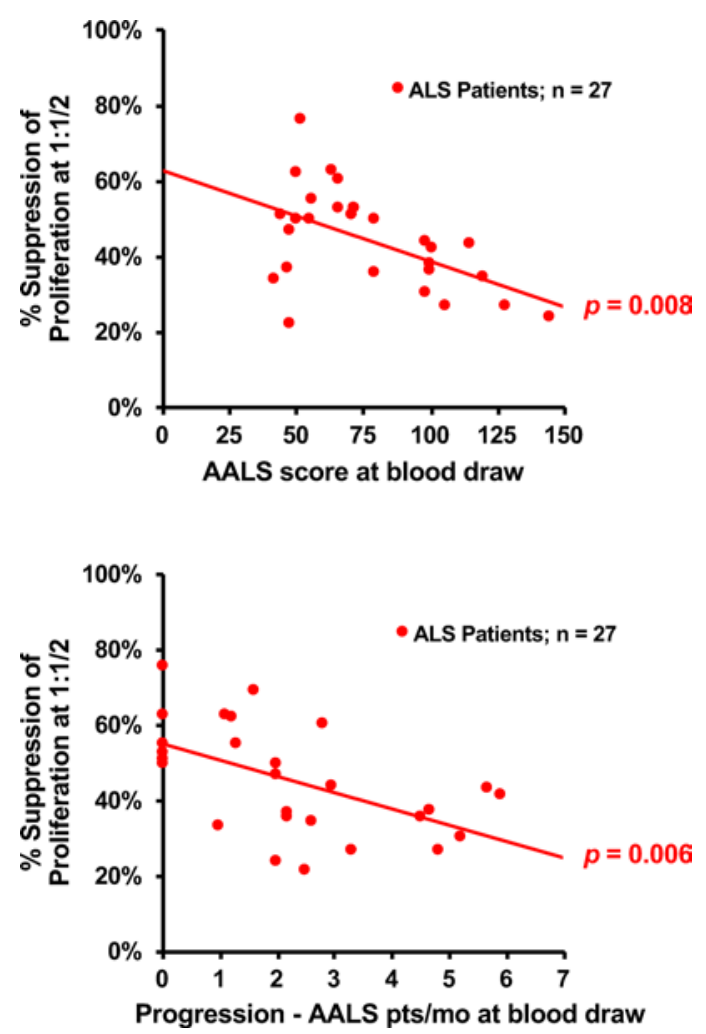


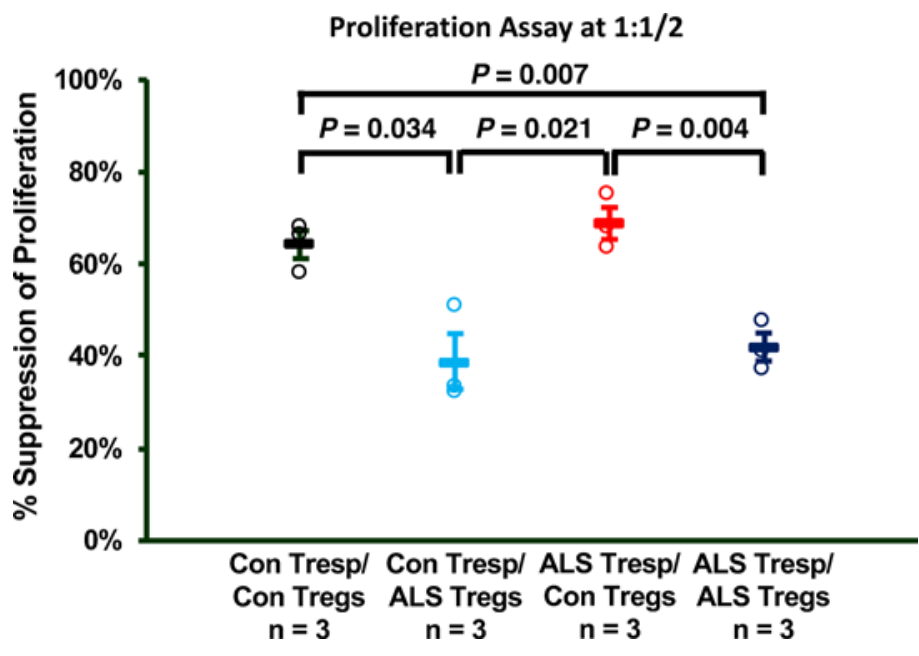

Figure 4. Dysfunction of ALS responder T lymphocytes (Tresps) versus control Tresps. Mixing studies demonstrated that when assayed at a 1:1/2 Tresp/Treg ratio, Tregs from patients with ALS could not suppress the proliferative response of target Tresps from either ALS patients or the controls (Con). Significance determined by Student's $t$ test.

impaired or the more rapidly progressing the ALS patient, the greater the Treg dysfunction.

ALS patients' Tresps are not dysfunctional. A critical question is whether the decreased suppressive capability of ALS Tregs was truly due to a decrease in the function of Tregs rather than an increase in the resistance of activated Tresps to Treg-induced inhibition. Thus, mixing experiments were performed in which Tregs from ALS patients and the control group were cocultured with their autologous target cells, or the converse, target cells isolated from either the control group or ALS patients. The Tresps from each patient with ALS and the healthy volunteer control expanded to a similar extent; thus, no inherent differences were seen in the expandability of the 2 Tresp populations between groups. When assayed at the 1:1/2 Tresp/Treg ratio, Tregs from ALS patients could not suppress the proliferative response of target Tresps from either ALS patients or the control group (suppression of $42 \%$ and 39\%, respectively; control Tresps/control Tregs vs. control Tresps/ALS Tregs, $P=$ 0.034; control Tresps/ALS Tregs vs. ALS Tresps/control Tregs, $P=0.021$; ALS Tresps/control Tregs vs. ALS Tresps/ALS Tregs, $P=0.004$; control Tresps/control Tregs vs. ALS Tresps/ALS Tregs, $P=0.007$; and control Tresps/control Tregs vs. ALS Tresps/control Tregs, $P=0.37$; Figure 4). In contrast, in the reciprocal experiments, Tregs from the control group suppressed the proliferative response of target Tresps derived from both control and ALS patients (suppression of $64 \%$ and $63 \%$, respectively). These data indicated that the primary regulatory defect was in the function of Tregs isolated from the circulation of ALS patients.

Epigenetic regulation of the FOXP3 locus. To determine whether the functionally distinct Tregs isolated from ALS patients exhibited unique FOXP3 epigenetic gene methylation patterns compared with Tregs from the control group, the methylation state of the region in the first intron of FOXP3, referred to as the Treg-specific demethylated region (TSDR), was investigated using the bisulphite sequencing technique. The level of Treg-specific demethylation has been shown to be a more specific and stable marker of human Tregs than FOXP3 mRNA expression. A caveat to this study was that the population of Tregs obtained from both ALS patients and the control group had a purity of $82 \%$ in comparison with the purity that could have been obtained with clonally derived Tregs. All $15 \mathrm{CpG}$ islands that were investigated exhibited more methylation in the TSDR of Tregs from rapidly progressing ALS patients compared with the TSDR of slowly progressing ALS patients and the control group (Figure 5A). The TSDR methylation was especially enhanced in islands 12 through 15 in the rapidly progressing ALS patients compared with slowly progressing ALS patients and the control group $(P<0.001$ rapidly progressing ALS patients vs. slowly progressing ALS patients or the control group). There were no differences across all $15 \mathrm{CpG}$ islands between slowly progressing ALS patients and the control. Overall, the TSDR of the Tregs from rapidly progressing patients was more methylated than the TSDRs of Tregs isolated from either slowly progressing ALS patients or the control group $(P<0.001$ and $P<0.001$, respectively; Figure 5B). Thus, the results related to decreased suppressive capacity of Tregs and methylation of the TSDR region in rapidly progressing patients as compared with slowly progressing patients, and with controls, was not due to an increase in contaminating $\mathrm{CD}^{+}{ }^{+} \mathrm{CD} 4^{+} \mathrm{CD} 25^{\mathrm{hi}} \mathrm{CD} 127^{+}$Tresps in the preparations.

In vitro expansion restores ALS patients' Tregs' suppressive abilities. It was also critical to determine whether Tregs from ALS patients were inherently dysfunctional, or whether they could regain their suppressive capabilities when removed from their endogenous environment and expanded in vitro. Circulating peripheral blood mononuclear cells (PBMCs) were obtained from patients with ALS and healthy controls, and then sent to a good manufacturing practice (GMP) facility, an FDA-mandated a system for ensuring that products are consistently produced and controlled according to quality standards. The PBMCs were enriched for $\mathrm{CD} 4^{+} \mathrm{T}$ lymphocytes by depleting $\mathrm{CD} 8^{+}$and $\mathrm{CD} 19^{+}$lymphocytes, and the $\mathrm{CD} 4^{+} \mathrm{CD} 25^{\mathrm{h}}$ Tregs were then enriched by positive selection; the majority of the $\mathrm{CD} 4^{+} \mathrm{CD} 25^{\text {hi }}$ Tregs expressed FOXP3. 

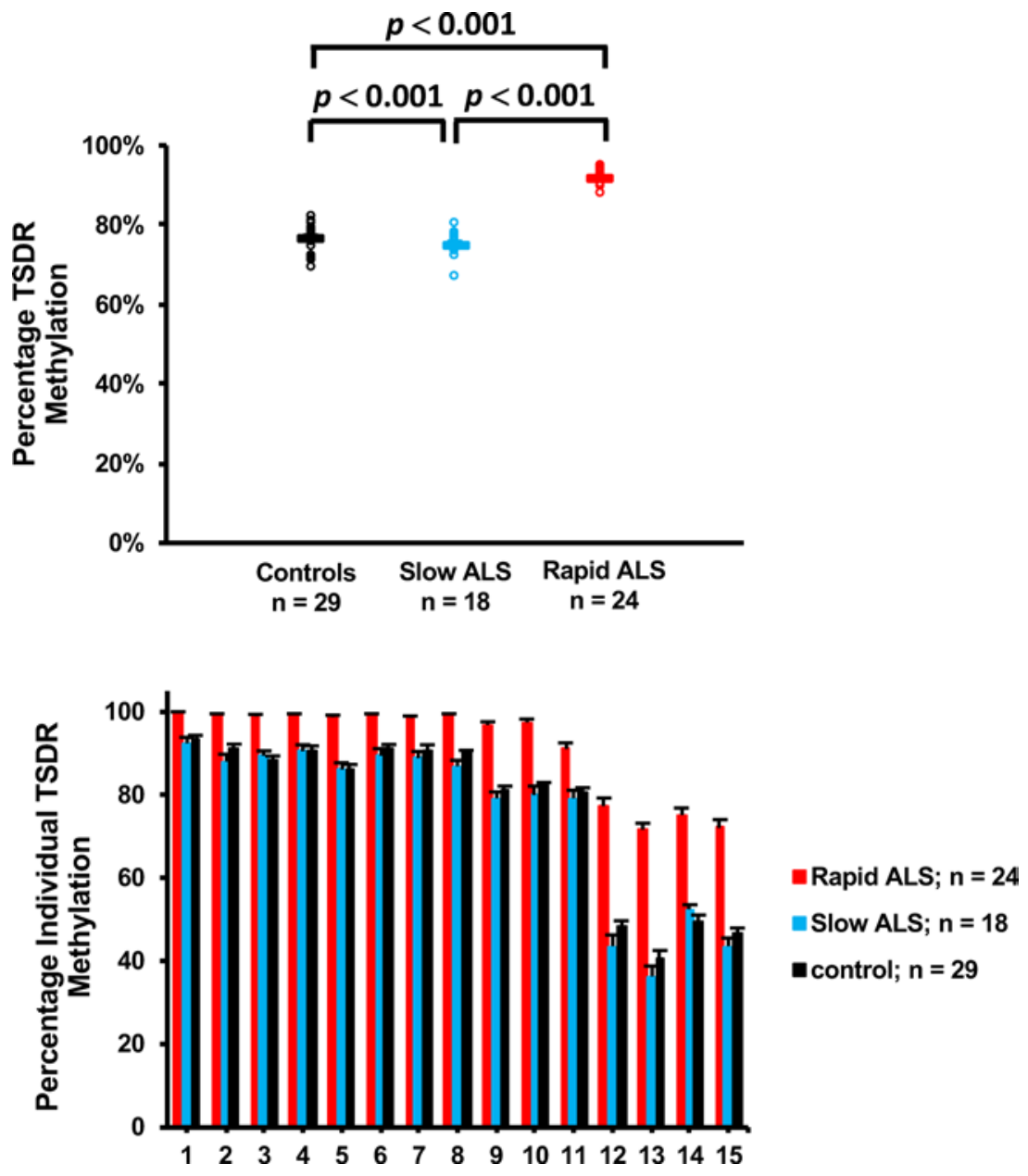

Figure 5. The percentage methylation of the Tregspecific demethylated region (TSDR) between ALS patients' and controls' Tregs. (A) The percentage TSDR methylation combining all $15 \mathrm{CpG}$ islands among the 3 groups. ALS patients exhibited more methylation in the TSDR of Tregs compared with the TSDR of slowly progressing ALS patients and the control group. Significance determined by Student's $t$ test. (B) The percentage TSDR methylation of each individual $\mathrm{CPC}$ island among the 3 groups. The TSDR methylation was especially enhanced in islands 12 through 15 in the rapidly progressing ALS patients compared with slowly progressing ALS patient and the control group. There were no differences across all $15 \mathrm{CpG}$ island between slowly progressing ALS patients and the control group.

Prior to expanding the Tregs, a portion of the cells were evaluated for their ability to suppress autologous Tresps. In confirmation of our prior data, the ALS patients' Tregs had less ability to suppress their corresponding autologous Tresps at the 1:1 Tresp/Treg ratio (Figure 6A). In contrast, at the 1:1 Tresp/Treg ratio, the control group's Tregs suppressed their corresponding autologous Tresps. Thus, at baseline, the suppressive function of the ALS patients' Tregs was impaired compared with the control group's Tregs.

Since we have previously demonstrated in mSOD $1^{\mathrm{G} 93 \mathrm{~A}}$ mice that passive transfers of Tregs only had an effect of slowing the rate of disease progression when the mSOD $1^{\mathrm{G} 93 \mathrm{~A}}$ mice were in the slowly progressing phase of their disease, we selected slowly progressing ALS for Treg expan-

sion studies; passive transfers of Tregs had no effect on slowing the rate of disease progression when the ALS mice were in the rapidly progressing phase of their disease $(3,4)$. Thus, the remaining portions of the age-matched ALS patients' and control group's Tregs were expanded in vitro in the presence of IL-2 and rapamycin, which preferentially expands functional Tregs while suppressing the growth of Tresps, for a period of 4 weeks. Following completion, which showed a mean 15-fold (7.5- to 21-fold) increase in the numbers of Tregs, the ALS patients' and control group's Tregs were again evaluated for their ability to suppress autologous Tresps. The suppressive activity of the healthy control was $48 \%$ pre-expansion ([1 $-(43.5 \% / 83.6 \%)] \times 100 \%)$ and $53.6 \%$ post-expansion. In contrast, the ALS-1 patient had $0 \%$ suppressive activity pre-expansion and $39.6 \%$ post-expansion. In the ALS- 2 and ALS-3 patients, the pre-expansion was $0 \%$ and $1.7 \%$, respectively, and $27.8 \%$ and 44.25 post-expansion, respectively. Thus, after expansion, the ALS patients' Tregs acquired the ability to suppress their corresponding autologous Tresps to nearly the same levels as observed with the control's Tregs at a 1:1 Tresp/Treg ratio (Figure 6B).

\section{Discussion}

Our focus on $\mathrm{T}$ lymphocyte responses was based on our earlier investigations in the $\mathrm{mSOD} 1$ transgenic model of ALS. The protective function of T lymphocytes was documented by the accelerated progression of disease and shortened survival of transgenic mice that overexpressed mSOD1 but lacked CD4 ${ }^{+} \mathrm{T}$ lymphocytes (19). Tregs were suggested to be the specific subpopulation mediating protection based on their increased levels during early slowly progressing stages of disease, and their decreased levels during late rapidly progressing stages. More definitive evidence for their protective function was the demonstration that passive transfer of Tregs into recipient ALS mice lengthened disease duration and prolonged survival (20).

Tregs were subsequently found to have a protective role in ALS patients: reduced Treg numbers and FOXP3 expression were associated with more rapid disease progression (37). Low circulating levels of 
A

Healthy Control

ALS-1

ALS-2

ALS-3
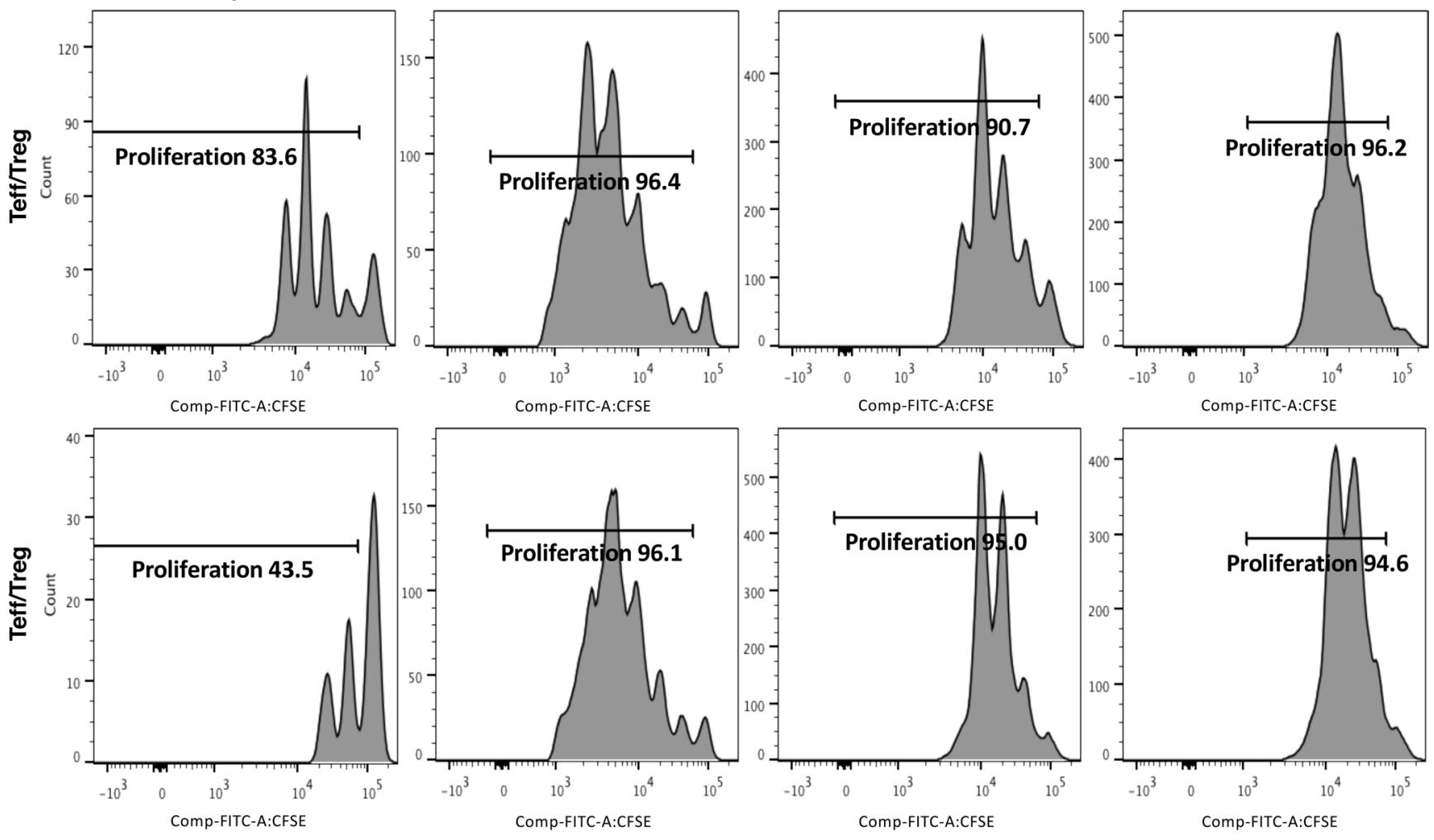

B

ALS-1

ALS-2
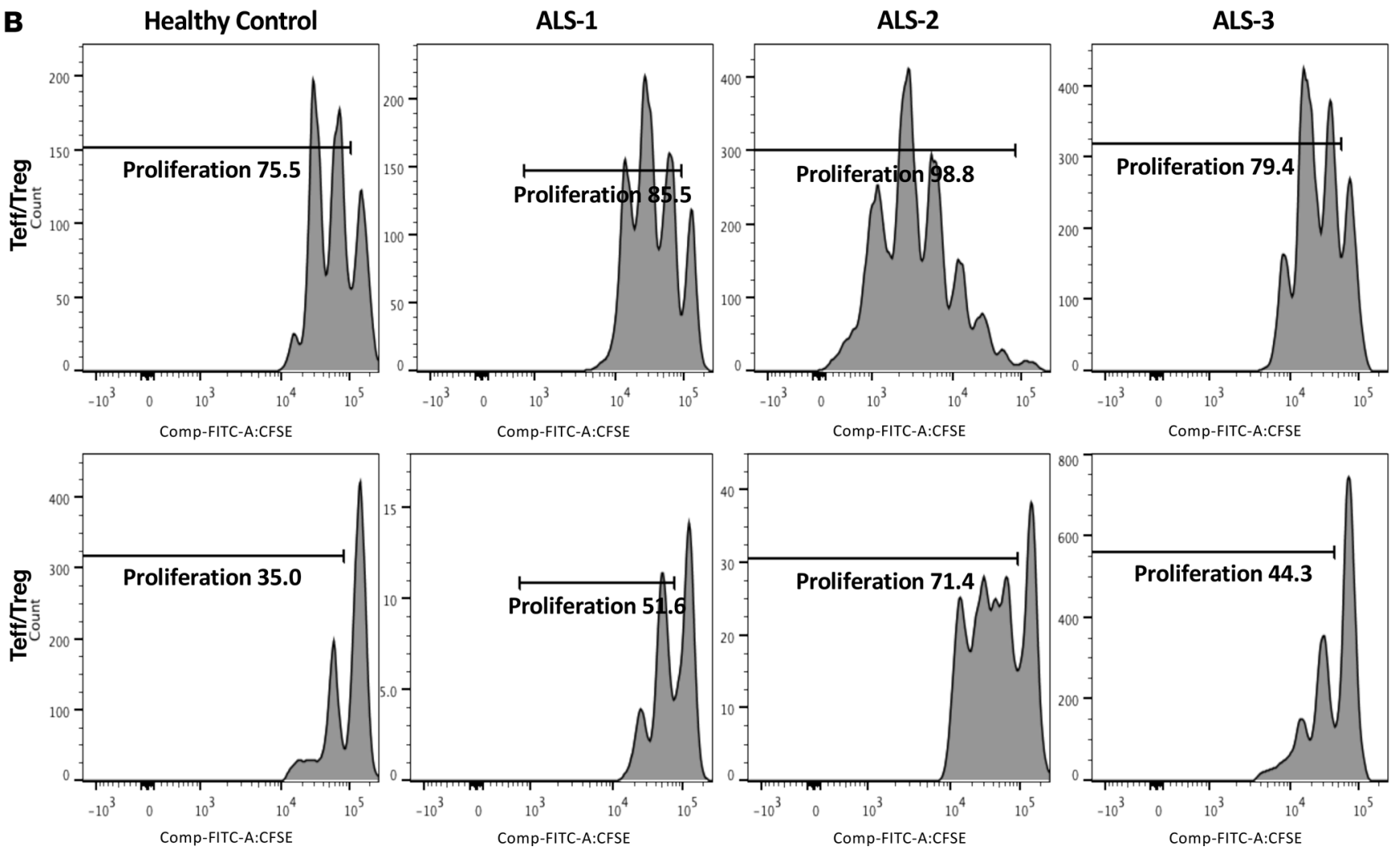

Figure 6. In vitro expansion induces a recovery of ALS Tregs' suppressive capacities. (A) Prior to expansion, ALS patients' Tregs were again found to be less capable of suppressing their corresponding autologous CD4+CD25- responder T lymphocytes (Tresps) at a 1:1 Tresp/Treg ratio than the control group's Tregs were of suppressing their corresponding autologous Tresps. (B) After expansion, the ALS patients' Tregs acquired the ability to suppress their corresponding autologous Tresps to nearly same degree as the control group's Tregs at a 1:1 Tresp/Treg ratio. 
Tregs and FOXP3 expression were associated with increased mortality after 3.5 years, while higher levels of Tregs and FOXP3 expression were associated with lower mortality over the same period of time. Thus, circulating Tregs influenced disease progression rates, and early reduced FOXP3 expression levels were indicative of progression rates at the time of blood collection, and predictive of future rapid progression and attenuated survival.

In the present study, we documented that circulating Tregs in patients with ALS were less effective in suppressing Tresp proliferation compared with Tregs obtained from healthy volunteers. The suppressive dysfunction was most marked in rapidly progressing patients, although Tregs from both slowly and rapidly progressing ALS patients were dysfunctional. It has been established that inappropriate $\mathrm{T}$ lymphocyte responses in the CNS affect the pathogenesis of a broad range of neuroinflammatory and neurodegenerative disorders. However, to our knowledge, this is the first report documenting the dysfunction of Tregs in patients with slowly or rapidly progressing ALS, compared with Tregs obtained from healthy volunteers. ALS is a devastating neurodegenerative disorder with differing rates of progression and lengths of disease. While some ALS patients progress rapidly and survive only a year or two after diagnosis, other patients progress slowly and can survive 4-6 years or more after diagnosis with quality of life for prolonged periods. The present study provides evidence of altered Treg responses in patients with ALS, with the variable Treg suppressive functions contributing to these diverse rates of progression. Furthermore, these results are not confounded by high numbers of contaminating $\mathrm{CD} 3^{+} \mathrm{CD} 4{ }^{+} \mathrm{CD} 25^{\mathrm{hi}} \mathrm{CD} 127^{+}$cells, which would have skewed the results; the population of $\mathrm{CD} 3^{+} \mathrm{CD} 4^{+} \mathrm{CD} 25^{\mathrm{hi}} \mathrm{CD} 127^{+}$cells was similar between patients with ALS and the samples obtained from healthy volunteers.

Tregs are cellular mediators of immunological tolerance that possess the capacity to suppress various types of immune responses. Active suppression by Tregs plays a key role in the control of self-antigen-reactive $\mathrm{T}$ lymphocytes and the induction of peripheral tolerance in vivo. Seminal experiments performed by Sakaguchi et al. showed that depletion of Tregs resulted in the onset of systemic autoimmune disease in mice (23-26). Furthermore, cotransfer of Tregs with Teffs prevented the development of experimentally induced autoimmune diseases such as colitis, gastritis, insulin-dependent autoimmune diabetes, and thyroiditis. Tregs are well-known immunoregulatory subsets that prevent activation and effector function of activated Tresps. Although autoreactive $\mathrm{T}$ lymphocytes are present in healthy individuals and patients with autoimmune disorders, autoreactive $\mathrm{T}$ lymphocytes found in patients with autoimmune diseases are more easily activated compared with those from healthy control volunteers.

Our recent study demonstrated that leukocytes obtained from patients with ALS at all stages of disease contained reduced numbers of $\mathrm{CD} 4{ }^{+} \mathrm{CD} 25^{+}$Tregs; the numbers of Tregs inversely correlated with disease progression rates (37). In rapidly progressing ALS patients, FOXP3 expression and fluorescence intensity of $\mathrm{CD}^{+} \mathrm{FOXP}^{+}$Tregs were reduced. The present study demonstrates that the Tregs are dysfunctional as well as being reduced in number. Tregs obtain immunosuppressive capacity by the upregulation of FOXP3, and persistent expression of this transcription factor is required to maintain their immune regulatory function and ensure immune homeostasis (25). To investigate the kinetics and function of Tregs, an earlier study used a mouse model of orthotopic corneal transplantation, the most common form of tissue grafting worldwide (40). Using in vitro functional assays and in vivo Treg adoptive transfer assays, this study showed that far more relevant than Treg frequency is their level of FOXP3 expression, which is directly associated with the potential of Tregs to prevent allograft rejection by producing regulatory cytokines and suppressing Teff activation.

The reduced intracellular FOXP3 protein expression in Tregs from patients with ALS was reflective of the FOXP3 mRNA expression in Tregs isolated from these patients. Rapidly progressing patients had less FOXP3 mRNA expression than slowly progressing patients and healthy volunteers. This confirms the earlier report demonstrating less FOXP3 in patients with ALS having a rapidly progressive disease (37). In addition, at the 1:1/2 Tresp/Treg ratio, the percentage of suppression was strongly correlated with FOXP3 expression; the rapidly progressing patients all fell below the ROC cutoff of 0.66 , again confirming the earlier report (37). Furthermore, at this same 1:1/2 Tresp/Treg ratio, the percentage of suppression was correlated with both the burden of disease as measured by the AALS score, and the rate of disease progression in all patients with ALS. Again, this result underscores the dysfunctional aspects of the Treg responses from patients with ALS.

A potential concern was that the population of Tregs obtained from both ALS patients and the control group had a purity of $82 \%$ in comparison with the purity that could have been obtained with clonally 
derived Tregs. Although studies in mice have shown FoxP3 to be a direct marker of Tregs, FOXP3 has also been shown to be transiently upregulated upon activation of human Tresps $(41,42)$. The upregulation of FOXP3 upon activation of Tresps in humans may result in an overestimation of Tregs. Even with the possible contamination of Tresps expressing FOXP3, we were still able to show that Tregs from patients with ALS were less suppressive compared with Tregs obtained from healthy volunteers. The more highly methylated TSDR of the rapidly progressing patients with ALS may aid in explaining why these cells were less suppressive than Tregs from either the slowly progressing patients with ALS or the healthy volunteer group. However, these data also suggested that the Tregs obtained from slowly progressing ALS patients were at least as suppressive on the proliferation of Tresps as the control group Tregs. Indeed, at the 1:1/8 and 1:1/4 CD $4^{+} \mathrm{CD} 25^{-}$Tresp/CD $4^{+} \mathrm{CD} 25^{\text {hi }}$ Treg ratios, Tregs from slowly progressing patients with ALS were able to suppress the proliferation of their autologous Tresps as efficiently as the Tregs obtained from the healthy volunteers suppressed their autologous Tresps.

Stable FOXP3 expression is achieved through epigenetic modification of the TSDR, an evolutionarily conserved, CpG-rich, noncoding element within the first intron of the FOXP3 gene locus $(43,44)$. The TSDR is demethylated only in Tregs stably expressing FOXP3 but is fully methylated in conventional CD4 ${ }^{+}$ $\mathrm{T}$ lymphocytes, and when generated in vitro, Tregs only transiently express FOXP3 $(43,45)$. To determine if the Tregs isolated from patients with ALS exhibited unique FOXP3 gene methylation patterns, the methylation state of the TSDR was examined using bisulphite sequencing. In the current study, the TSDR was determined to be more methylated in patients with ALS compared with the healthy volunteers. All 15 CpG islands exhibited more methylation in the TSDR of Tregs from patients with ALS having a rapidly progressing disease compared with the TSDR of patients with ALS having a slowly progressing disease. It is important to stress that these results were not influenced by the numbers of contaminating $\mathrm{CD}^{+} \mathrm{CD} 4{ }^{+} \mathrm{CD} 25^{\mathrm{hi}} \mathrm{CD} 127^{+}$cells because similar numbers of such cells were present in patients with ALS and the healthy volunteers.

It was also important to examine whether the loss of regulatory function could be explained by an increased resistance of activated Tresps to inhibition rather than decreased Treg function. To clarify this issue, we carried out mixing experiments in which patient and control Tregs were cocultured with the autologous and the converse target cells isolated from either healthy volunteers or patients with ALS. Tregs from patients with ALS were less effective in suppressing the proliferative response of target Tresps from either patients or healthy volunteers. In contrast, in the reciprocal experiments, Tregs from healthy volunteers suppressed the proliferative response of target Tresps derived from both volunteers and patients with ALS. These data indicate that the primary regulatory defect is in the function of Tregs isolated from the circulation of patients with ALS. Thus, the defect lies in the Treg function, as opposed to enhanced Tresp resistance in patients with ALS.

It was also important to determine if the loss of suppressive capabilities of the Tregs from patients with ALS was a permanent loss or if the suppressive function could be restored. Inappropriate $\mathrm{T}$ lymphocyte responses in the CNS affect the pathogenesis of a broad range of neuroinflammatory and neurodegenerative disorders. An earlier study reported a reduction in the functions of Tregs from patients with multiple sclerosis (MS), an autoimmune disease, compared with healthy volunteers (46). These data were among the first to demonstrate a functional defect in Tregs in a human autoimmune disease, and suggested that impaired suppression of Tresp proliferation and function could contribute to disease progression. Our own studies in the mouse model of ALS suggested that Tregs might influence disease progression by modulating the activation state of microglia, as well as by suppressing Teffs $(18,19)$. At early stages, increased Tregs were associated with increased IL-4 expression and levels of protective M2 microglia. At later rapidly progressing stages of disease, the decreased numbers of Tregs and the loss of FoxP3 were associated with decreased IL-4 expression, and increased expression of M1 microglial proinflammatory cytokines. Activated microglia increased with disease progression through the end stage as numbers of Tregs decreased (4, 5). The passive transfer of endogenous Tregs from ALS mice restored IL-4 levels and markedly decreased microglial proinflammatory cytokines, prolonging disease duration and survival (20).

The mechanism of Treg suppression of activated microglia in models of ALS was delineated with in vitro studies (47). When cocultured with mSOD1 adult microglia, mSOD1 Tregs suppressed the cytotoxic microglial factors NOX2 and iNOS through secretion of IL-4, and was not dependent on CTLA-4 engagement or release of IL-10 or TGF- $\beta$. Furthermore, IL- 4 inhibitory antibodies blocked the suppressive function of mSOD1 Tregs, and conditioned media from mSOD1 Tregs or the addition of IL- 4 reduced 
microglial NOX2 expression. Thus, Tregs, and more specifically CD4 ${ }^{+} \mathrm{CD} 25^{\text {hi }}$ Foxp $3^{+} \mathrm{IL}-4^{+}$Tregs, can suppress neurotoxic microglial activation and augment survival of mSOD1 mice. Since Tregs directly promote the antiinflammatory alternative M2 activation state of macrophages and microglia (29-33), dysfunctional Tregs would be anticipated to lose protective functions and enhance the proinflammatory state of macrophages and microglia. The pathogenic contribution of microglia was established by replacing mSOD1 CNS microglia with WT microglia or removing mSOD1 from microglia, resulting in prolonged survival of ALS mice $(18,21)$. Thus, there is an interdependence between the innate and adaptive immune systems.

In the mSOD1 transgenic mouse model of ALS, the mechanism of Treg suppression of Tresps differed from the Treg suppression of activated microglia. IL-4 was insufficient to promote suppression of Tresp proliferation. WT Tregs, and mSOD1 Tregs from 100- and 160-day-old mice, were able to inhibit proliferation and cytotoxicity of their respective Tresps. The inhibitory capacity of 100-day-old mSOD1 Tregs on 100 -day-old mSOD1 Tresps was mediated by the combination of IL-4, IL-10, and TGF- $\beta$. However, combination of blocking antibodies against IL-4, IL-10, and TGF- $\beta$ did not alter the Treg inhibition of Tresps isolated from WT or 160-day-old mSOD1 mice, suggesting that WT or 160-day-old Tregs suppress Tresp function through cell-cell contact or other mechanisms. Therefore, multiple mechanisms operate in Tregmediated suppression of Tresps and various molecules may contribute to Tresp suppression; a particular mechanism may play a dominant role under a particular condition, with different mechanisms operating in differing situations (48).

We also investigated whether the suppressive capabilities of the Tregs from patients with ALS were permanently altered or if the suppressive functions could be restored. When dysfunctional Tregs from patients with ALS were expanded in vitro in the presence of IL-2 and rapamycin, the latter of which preferentially expands functional Tregs while suppressing the growth of Tresps, the suppressive capacity of the Tregs from patients with ALS were restored and able to suppress their corresponding autologous Tresps to nearly the same levels as observed with healthy volunteer Tregs and their autologous Tresps. Thus, this finding demonstrates that the loss of suppressive capabilities of the Tregs from patients with ALS is not irreversible and can be restored. This also demonstrates the possible presence of a circulating or tissue factor in the blood of patients with ALS that actively inhibits the suppressive function of Tregs. However, it is also possible that expansion of ALS Tregs over a 4-week period, especially in the presence of rapamycin, selectively expands non-dysfunctional Tregs, and that the most impaired Tregs were not capable of expansion. This latter explanation may support the incomplete restoration of suppressive activity when compared with expanded Tregs from healthy volunteer controls.

These immune responses may occur within or outside the CNS, and the migration of antigen-specific $\mathrm{CD}^{+} \mathrm{T}$ lymphocytes from the periphery to the CNS can affect the inflammatory responses of resident glial cells as well as neuronal survival. These responses can either exacerbate neurotoxic responses or enhance neuroprotection (49). The specific destructive or protective mechanisms of these interactions are possibly linked to the relative numerical and functional dominance of Tregs, and their immunomodulatory effects on Teffs and macrophages/microglia populations in vivo. Furthermore, the altered immunomodulatory effects of Tregs from patients with ALS do not necessarily depend upon effects within the CNS. Motoneuron axons, axon terminals, and the neuromuscular junction are outside the blood-brain barrier, and are readily accessible to circulating innate and adaptive immune cells. In animal models of ALS, the presence of monocytes/macrophages surrounding the degenerating peripheral nerve fibers is a clear and early event that occurs prior to the onset of clinical signs of motor weakness, and is associated with a "dying back phenomenon" (50). In patients with ALS, a distal axonopathy is also a prominent pathological finding. Thus, the circulating dysfunctional Tregs in ALS could mediate effects peripherally, and need not enter the CNS to influence disease progression.

As mentioned earlier, a prior study demonstrated that patients with MS also have a loss of functional suppression of their Tregs (46). Tregs from the peripheral blood of MS patients had reduced suppressive function compared with the healthy volunteers. Although ALS is not an immune-initiated disease, but rather a chronic neurodegenerative disorder that is clearly modulated by both innate and the adaptive immune systems, these is also a suppressive dysfunction of Tregs playing a prominent role. Type 1 diabetes mellitus (T1D) is another immune disease that occurs in genetically susceptible individuals. Patients with T1D have also been shown to have dysfunctional Tregs (51). A phase 1 clinical trial to assess the safety of Treg adoptive immunotherapy in T1D demonstrated that ex vivo-expanded autologous and adoptively transferred $\mathrm{CD} 4^{+} \mathrm{CD} 127^{10 /-} \mathrm{CD} 25^{+}$Tregs were long lived, with up to $25 \%$ of 
the peak level remaining in the circulation 1 year after transfer. Additional immune studies showed transient increases in Tregs in recipients that retained a broad Treg FOXP ${ }^{+} \mathrm{CD} 4{ }^{+} \mathrm{CD} 25^{\mathrm{hi}} \mathrm{CD} 127^{\mathrm{lo}}$ phenotype long term. There were no infusion reactions or cell-therapy-related high-grade adverse events. These results support the development of a phase 2 trial to test the efficacy of the Treg therapy in patients with T1D. Again, although not a neurodegenerative disease, but an autoimmune disease, patients with T1D also have dysfunctional Tregs.

These data indeed suggest that ex vivo Tregs from patients with ALS lack suppressive capabilities compared with Tregs from healthy volunteers, and that the lack of suppression correlates with disease burden and rates of progression in these ALS patients. Thus, the ability of the ALS dysfunctional Tregs to regain their suppressive capabilities following removal from their environment and expansion in vitro suggests a novel therapeutic strategy for ALS patients. Since there are no meaningful therapies presently available for ALS, autologous transfer of expanded Tregs represents a promising approach for slowing disease progression in patients with ALS, as they did in transgenic animal models of ALS.

\section{Methods}

Isolation of Tregs and Teffs. After receiving IRB approval and written informed consent from patients diagnosed with definite or probable sporadic ALS according to revised El Escorial criteria of the World Federation of Neurology (39), peripheral blood was drawn and processed for isolation of Tregs and Teffs. Briefly, the isolation of $\mathrm{CD} 4{ }^{+} \mathrm{CD} 25^{+}$Tregs was performed in a 2 -step procedure using a $\mathrm{CD} 4{ }^{+} \mathrm{CD} 25^{+}$ Regulatory $\mathrm{T}$ Cell Isolation Kit (Miltenyi Biotec). First, the non-CD4+ cells were indirectly magnetically labeled with a cocktail of biotin-conjugated antibodies. Then the $\mathrm{CD} 4^{+} \mathrm{CD} 25^{+}$Tregs were directly labeled with $\mathrm{CD} 25^{+}$MicroBeads and isolated by positive selection over a MACS column, which was placed in the magnetic field of a MACS separator. After removing the column from the magnetic field, the magnetically retained $\mathrm{CD} 4{ }^{+} \mathrm{CD} 25^{+}$Tregs were eluted as a positively selected cell fraction. To increase the purity, the positively selected cell fraction was run in a second column. The isolated Tregs and Teffs were then cocultured at different ratios in the presence of a polyclonal stimulus using Miltenyi Biotec's Human Treg Suppression Inspector kit. When cultured alone, Tregs showed a hypoproliferative anergic response. In contrast, Teffs alone showed a proliferative response. Coculture of Tregs with Teffs at ratios ranging from 1:1 to 8:1 (Teffs/Tregs) resulted in reduced proliferation of Teffs as determined by ${ }^{3} \mathrm{H}$-thymidine incorporation. We also performed mixing experiments in which patient and control CD $4^{+} \mathrm{CD} 25^{\mathrm{h}}$ Tregs cells were cocultured with the autologous and the converse target cells isolated from either healthy control volunteers or ALS patients. Mixing experiments were performed in the presence of fixed accessory cells autologous to the $\mathrm{CD} 4^{+} \mathrm{CD} 25^{-}$responder Teffs.

Flow cytometry. Multicolor flow cytometry was used to assess the phenotype of $\mathrm{CD} 4^{+} \mathrm{CD} 25^{+}$Tregs. mAbs against the following surface molecules were used: CD3 BV650 (catalog 563851), CD8 BV450 (catalog 561426), CD4 APC-H7 (catalog 560837), CD25 PE (catalog 560989), and Live Dead Aqua (Molecular Probes, L34957). For intracellular staining, cells were fixed and permeabilized using the FoxP3/Transcription Factor Staining Buffer Set (eBioscience, 00-5523) following the manufacturer's instructions, and then stained with FoxP3 PE-Cy5.5 (eBioscience, catalog 35-4776). Cells were acquired on the LSRFortessa cytometer (BD Biosciences), and data were analyzed using FlowJo software (TreeStar).

PBMCs were depleted of CD8 ${ }^{+} \mathrm{T}$ lymphocytes and $\mathrm{CD} 19^{+} \mathrm{B}$ lymphocytes using GMP-grade CD8 and CD19 microbeads (Miltenyi Biotec). The CD8/CD19-depleted fraction was then incubated with GMP-grade CD25 microbeads (Miltenyi Biotec), to enrich for $\mathrm{CD} 4{ }^{+} \mathrm{CD} 25^{+} \mathrm{T}$ lymphocytes, following the manufacturer's instructions. All selection steps were performed manually using LS separation columns (Miltenyi Biotec). To isolate Tregs from the leukapheresis products, we used the depletion and enrichment tubing sets from CliniMACS (Miltenyi Biotec).

Bisulphite sequencing. Genomic DNA from isolated Tregs was extracted with the QIAamp DNA Blood Mini Kit (Qiagen) following the supplier's recommendations. Sodium bisulphite treatment of genomic DNA was performed according to Olek et al. (52). A minimum of $60 \mathrm{ng}$ bisulfite-treated (EpiTect; Qiagen) genomic DNA was used in a real-time PCR to quantify the FoxP3 TSDR. Real-time PCR was performed in a final reaction volume of $20 \mu$ containing $10 \mu$ FastStart Universal Probe Master (Roche Diagnostics), $50 \mathrm{ng} / \mu 1$ lambda DNA (New England Biolabs), 5 pmol/ $\mu 1$ methylation or non-methylation-specific probe, $30 \mathrm{pmol} / \mu 1$ methylation or non-methylation-specific primers, and 60 ng bisulfite-treated DNA or an equivalent amount of plasmid standard. The samples were analyzed in 
triplicate on an ABI 7500 cycler (Life Technologies). In PCR amplification, uracils were replicated as thymidines. Thus, detection of a cytosine in sequencing reactions reflects methylation of the genomic DNA at that site. Detection of a thymidine at the same site reflects instead the absence of a methyl modification of the genomic cytosine.

Expansion of Tregs. Bead-selected $\mathrm{CD} 4{ }^{+} \mathrm{CD} 25^{+} \mathrm{T}$ lymphocytes were suspended at a concentration of $0.5 \times 10^{6} \mathrm{cells} / \mathrm{ml}$ in TexMACS GMP media (Miltenyi Biotec) in the presence or absence of $100 \mathrm{nM}$ of GMP-grade rapamycin (Miltenyi Biotec) and stimulated with MACS GMP ExpAct Treg Kit (Miltenyi Biotec) at a 4:1 bead-to-cell ratio. Six days later, $500 \mathrm{IU} / \mathrm{ml}$ of clinical grade human recombinant IL-2 (Proleukin; Chiron) was added to the culture. Fresh media containing rapamycin and IL-2 were added to the cells every 2 to 3 days. After 2 weeks of culture, cells were harvested, washed, and restimulated with the MACS GMP ExpAct Treg Kit at a 1:1 bead-to-cell ratio in the presence of rapamycin and IL-2. Fresh media containing rapamycin and IL-2 were replenished every 2 to 3 days. After 25 days of culture, cells were harvested and characterized.

Treg suppression assays. To determine the ability of Tregs to suppress the proliferation of Teffs in vitro, CFSE-stained (Life Technologies) $\mathrm{CD}^{+} \mathrm{CD} 25^{-} \mathrm{T}$ lymphocytes were stimulated for 3 to 5 days with anti-CD3/28 Dynabeads (Invitrogen) at 1:1 cell-to-bead ratio and cultured in the presence or absence of Tregs. Cells were harvested, washed, and stained for surface markers and CFSE dilution was used as a measure of cell proliferation. Samples were acquired on the FACSFortessa and data were analyzed using FlowJo software.

Statistics. A mixed-effects linear model was utilized to evaluate the effects of group and ratio on percentage suppression, which was the response variable. Fixed factors included ratio (treated as equally spaced, ordered categories), group (rapidly progressing ALS patients, slowly progressing ALS patients, or healthy control volunteers), and the interaction between ratio and group. 95\% CIs were calculated. A random intercept and slope for each patient was modeled. Since all values at the ratio 1:0 were 0 , this measurement point was omitted from the model. For other comparisons, an ANOVA or Student's $t$ test was performed. Data are expressed as mean \pm SEM and $P$ values less than 0.05 were considered significant.

\section{Author contributions}

DRB, KR, EJS, and SHA designed the study. DRB, WZ, JW, XZ, SW, JRT, and ASA conducted the experiments. DRB, SHA, and DN analyzed the data. DRB and SHA wrote the manuscript.

\section{Acknowledgments}

This study was funded by the National Institute of Neurological Disorders and Stroke (R21NS07994301A1) and the Muscular Dystrophy Association (MDA295199). We are grateful to Yuanfang Liu for her technical assistance.

This manuscript is dedicated to our colleague and friend J.S. Henkel.

Address correspondence to: Stanley H. Appel, Department of Neurology, Houston Methodist Neurological Institute, 6560 Fannin Street, Suite ST-802, Houston, Texas 77030, USA. Phone: 713.441.3765; E-mail: sappel@houstonmethodist.org.

1. Zhao W, Beers DR, Appel SH. Immune-mediated mechanisms in the pathoprogression of amyotrophic lateral sclerosis. $J$ Neuroimmune Pharmacol. 2013;8(4):888-899.

2. Appel SH, Beers DR, Henkel JS. T cell-microglial dialogue in Parkinson's disease and amyotrophic lateral sclerosis: are we listening? Trends Immunol. 2010;31(1):7-17.

3. Henkel JS, et al. Presence of dendritic cells, MCP-1, and activated microglia/macrophages in amyotrophic lateral sclerosis spinal cord tissue. Ann Neurol. 2004;55(2):221-235.

4. Henkel JS, Beers DR, Siklós L, Appel SH. The chemokine MCP-1 and the dendritic and myeloid cells it attracts are increased in the mSOD1 mouse model of ALS. Mol Cell Neurosci. 2006;31(3):427-437.

5. Henkel JS, Beers DR, Zhao W, Appel SH. Microglia in ALS: the good, the bad, and the resting. J Neuroimmune Pharmacol. 2009;4(4):389-398.

6. Graves MC, et al. Inflammation in amyotrophic lateral sclerosis spinal cord and brain is mediated by activated macrophages, mast cells and T cells. Amyotroph Lateral Scler Other Motor Neuron Disord. 2004;5(4):213-219.

7. Kawamata T, Akiyama H, Yamada T, McGeer PL. Immunologic reactions in amyotrophic lateral sclerosis brain and spinal cord tissue. Am J Pathol. 1992;140(3):691-707. 
8. Lampson LA, Kushner PD, Sobel RA. Major histocompatibility complex antigen expression in the affected tissues in amyotrophic lateral sclerosis. Ann Neurol. 1990;28(3):365-372.

9. Troost D, van den Oord JJ, de Jong JM, Swaab DF. Lymphocytic infiltration in the spinal cord of patients with amyotrophic lateral sclerosis. Clin Neuropathol. 1989;8(6):289-294.

10. Troost D, Van den Oord JJ, Vianney de Jong JM. Immunohistochemical characterization of the inflammatory infiltrate in amyotrophic lateral sclerosis. Neuropathol Appl Neurobiol. 1990;16(5):401-410.

11. Engelhardt JI, Tajti J, Appel SH. Lymphocytic infiltrates in the spinal cord in amyotrophic lateral sclerosis. Arch Neurol. 1993;50(1):30-36.

12. Lincecum JM, et al. From transcriptome analysis to therapeutic anti-CD40L treatment in the SOD1 model of amyotrophic lateral sclerosis. Nat Genet. 2010;42(5):392-399.

13. Mantovani S, et al. Immune system alterations in sporadic amyotrophic lateral sclerosis patients suggest an ongoing neuroinflammatory process. J Neuroimmunol. 2009;210(1-2):73-79.

14. Seksenyan A, et al. Thymic involution, a co-morbidity factor in amyotrophic lateral sclerosis. J Cell Mol Med. 2010;14(10):2470-2482.

15. Shi N, et al. Increased IL-13-producing T cells in ALS: positive correlations with disease severity and progression rate. $J$ Neuroimmunol. 2007;182(1-2):232-235.

16. Zhang R, et al. Evidence for systemic immune system alterations in sporadic amyotrophic lateral sclerosis (sALS). $J$ Neuroimmunol. 2005;159(1-2):215-224.

17. Banerjee R, et al. Adaptive immune neuroprotection in G93A-SOD1 amyotrophic lateral sclerosis mice. PLoS One. 2008;3(7):e2740.

18. Beers DR, et al. Wild-type microglia extend survival in PU.1 knockout mice with familial amyotrophic lateral sclerosis. Proc Natl Acad Sci USA. 2006;103(43):16021-16026.

19. Beers DR, Henkel JS, Zhao W, Wang J, Appel SH. CD4 ${ }^{+}$T cells support glial neuroprotection, slow disease progression, and modify glial morphology in an animal model of inherited ALS. Proc Natl Acad Sci USA. 2008;105(40):15558-15563.

20. Beers DR, et al. Endogenous regulatory $\mathrm{T}$ lymphocytes ameliorate amyotrophic lateral sclerosis in mice and correlate with disease progression in patients with amyotrophic lateral sclerosis. Brain. 2011;134(Pt 5):1293-1314.

21. Boillée S, Vande Velde C, Cleveland DW. ALS: a disease of motor neurons and their nonneuronal neighbors. Neuron. 2006;52(1):39-59.

22. Chiu IM, et al. T lymphocytes potentiate endogenous neuroprotective inflammation in a mouse model of ALS. Proc Natl Acad Sci USA. 2008;105(46):17913-17918.

23. Sakaguchi S, Fukuma K, Kuribayashi K, Masuda T. Organ-specific autoimmune diseases induced in mice by elimination of $\mathrm{T}$ cell subset. I. Evidence for the active participation of T cells in natural self-tolerance; deficit of a $\mathrm{T}$ cell subset as a possible cause of autoimmune disease. J Exp Med. 1985;161(1):72-87.

24. Sakaguchi S. Naturally arising Foxp3-expressing $\mathrm{CD} 25^{+} \mathrm{CD} 4^{+}$regulatory $\mathrm{T}$ cells in immunological tolerance to self and non-self Nat Immunol. 2005;6(4):345-352.

25. Sakaguchi S, Yamaguchi T, Nomura T, Ono M. Regulatory T cells and immune tolerance. Cell. 2008;133(5):775-787.

26. Sakaguchi S, Miyara M, Costantino CM, Hafler DA. FOXP3 ${ }^{+}$regulatory T cells in the human immune system. Nat Rev Immunol. 2010;10(7):490-500.

27. Reynolds AD, Banerjee R, Liu J, Gendelman HE, Mosley RL. Neuroprotective activities of CD $4^{+} \mathrm{CD} 25^{+}$regulatory T cells in an animal model of Parkinson's disease. J Leukoc Biol. 2007;82(5):1083-1094.

28. Reynolds AD, Stone DK, Hutter JA, Benner EJ, Mosley RL, Gendelman HE. Regulatory T cells attenuate Th17 cell-mediated nigrostriatal dopaminergic neurodegeneration in a model of Parkinson's disease. J Immunol. 2010;184(5):2261-2271.

29. Tiemessen MM, Jagger AL, Evans HG, van Herwijnen MJ, John S, Taams LS. CD $4^{+} C D 25^{+}$Foxp3 $3^{+}$regulatory T cells induce alternative activation of human monocytes/macrophages. Proc Natl Acad Sci USA. 2007;104(49):19446-19451.

30. Li M, Lin J, Wang Z, He S, Ma X, Li D. Oxidized low-density lipoprotein-induced proinflammatory cytokine response in macrophages are suppressed by $\mathrm{CD} 4 \mathrm{CD} 25(+)$ Foxp3(+) regulatory $\mathrm{T}$ cells through downregulating toll like receptor 2-mediated activation of NF-kappaB. Cell Physiol Biochem. 2010;25(6):649-656.

31. Liu G, et al. Phenotypic and functional switch of macrophages induced by regulatory $\mathrm{CD} 4{ }^{+} \mathrm{CD} 25^{+} \mathrm{T}$ cells in mice. Immunol Cell Biol. 2011;89(1):130-142.

32. Mahnke K, Bedke T, Enk AH. Regulatory conversation between antigen presenting cells and regulatory T cells enhance immune suppression. Cell Immunol. 2007;250(1-2):1-13.

33. Reynolds AD, Stone DK, Mosley RL, Gendelman HE. Proteomic studies of nitrated alpha-synuclein microglia regulation by CD4 ${ }^{+}$CD $25^{+}$T cells. J Proteome Res. 2009;8(7):3497-3511.

34. Reynolds AD, Stone DK, Mosley RL, Gendelman HE. Nitrated \{alpha\}-synuclein-induced alterations in microglial immunity are regulated by CD4 ${ }^{+} \mathrm{T}$ cell subsets. J Immunol. 2009;182(7):4137-4149.

35. Cools N, et al. Immunosuppression induced by immature dendritic cells is mediated by TGF-beta/IL-10 double-positive CD4 regulatory T cells. J Cell Mol Med. 2008;12(2):690-700.

36. Savage ND, et al. Human anti-inflammatory macrophages induce Foxp $3^{+} \mathrm{GITR}^{+} \mathrm{CD} 25^{+}$regulatory T cells, which suppress via membrane-bound TGFbeta-1. J Immunol. 2008;181(3):2220-2226.

37. Henkel JS, et al. Regulatory T-lymphocytes mediate amyotrophic lateral sclerosis progression and survival. EMBO Mol Med. 2013;5(1):64-79.

38. Alsuliman A, et al. A robust, good manufacturing practice-compliant, clinical-scale procedure to generate regulatory $\mathrm{T}$ cells from patients with amyotrophic lateral sclerosis for adoptive cell therapy. Cytotherapy. 2016;18(10):1312-1324.

39. Brooks BR. El Escorial World Federation of Neurology criteria for the diagnosis of amyotrophic lateral sclerosis. Subcommittee on Motor Neuron Diseases/Amyotrophic Lateral Sclerosis of the World Federation of Neurology Research Group on Neuromuscular Diseases and the El Escorial "Clinical limits of amyotrophic lateral sclerosis" workshop contributors. J Neurol Sci. 1994;124 Suppl:96-107.

40. Chauhan SK, Saban DR, Lee HK, Dana R. Levels of Foxp3 in regulatory T cells reflect their functional status in transplantation. 
J Immunol. 2009;182(1):148-153.

41. Siewert C, et al. Experience-driven development: effector/memory-like alphaE ${ }^{+}$Foxp $3^{+}$regulatory $\mathrm{T}$ cells originate from both naive T cells and naturally occurring naive-like regulatory T cells. J Immunol. 2008;180(1):146-155.

42. Allan SE, et al. Activation-induced FOXP3 in human T effector cells does not suppress proliferation or cytokine production. Int Immunol. 2007;19(4):345-354.

43. Schreiber L, et al. The Treg-specific demethylated region stabilizes Foxp3 expression independently of NF-кB signaling. PLoS One. 2014;9(2):e88318.

44. Huehn J, Polansky JK, Hamann A. Epigenetic control of FOXP3 expression: the key to a stable regulatory T-cell lineage? Nat Rev Immunol. 2009;9(2):83-89.

45. Polansky JK, et al. DNA methylation controls Foxp3 gene expression. Eur J Immunol. 2008;38(6):1654-1663.

46. Viglietta V, Baecher-Allan C, Weiner HL, Hafler DA. Loss of functional suppression by CD $4^{+} \mathrm{CD} 25^{+}$regulatory T cells in patients with multiple sclerosis. J Exp Med. 2004;199(7):971-979.

47. Zhao W, Beers DR, Liao B, Henkel JS, Appel SH. Regulatory T lymphocytes from ALS mice suppress microglia and effector T lymphocytes through different cytokine-mediated mechanisms. Neurobiol Dis. 2012;48(3):418-428.

48. Sakaguchi S, Wing K, Onishi Y, Prieto-Martin P, Yamaguchi T. Regulatory T cells: how do they suppress immune responses? Int Immunol. 2009;21(10):1105-1111.

49. Anderson KM, Olson KE, Estes KA, Flanagan K, Gendelman HE, Mosley RL. Dual destructive and protective roles of adaptive immunity in neurodegenerative disorders. Transl Neurodegener. 2014;3(1):25

50. Fischer LR, et al. Amyotrophic lateral sclerosis is a distal axonopathy: evidence in mice and man. Exp Neurol. 2004;185(2):232-240.

51. Bluestone JA, et al. Type 1 diabetes immunotherapy using polyclonal regulatory T cells. Sci Transl Med. 2015;7(315):315ra189.

52. Olek A, Oswald J, Walter J. A modified and improved method for bisulphite based cytosine methylation analysis. Nucleic Acids Res. 1996;24(24):5064-5066. 